\title{
El COVID-19 también Afecta el Sistema Nervioso por una de sus Compuertas: El Órgano Vascular de la Lámina Terminal y el Nervio Olfatorio. Alerta Neurológica, Prueba de Disosmia o Anosmia Puede Ayudar a Un Diagnóstico Rápido
}

COVID-19 also Affects the Nervous System by One of its Gates: The Vascular Organ of Lamina Terminalis and the Olfactory Nerve. Neurological Alert, Dysosmia or Anosmia Test Can Help to A Quick Diagnosis

Jorge Eduardo Duque Parra ${ }^{1,2,3}$; Daniela Duque Montoya ${ }^{3}$ \& Félix John César Peláez ${ }^{1,4}$

DUQUE PARRA, J. E.; DUQUE MONTOYA, D. \& PELÁEZ, F. J. C. EI COVID-19 también afecta el sistema nervioso por una de sus compuertas: el órgano vascular de la lámina terminal y el nervio olfatorio. Alerta neurológica, prueba de disosmia o anosmia puede ayudar a un diagnóstico rápido. Int. J. Odontostomat., 14(3):285-287, 2020.

RESUMEN: Se presenta una breve revisión de órgano vascular de la lámina terminal (organum vasculosum laminae terminalis) y el nervio olfatorio, el primero un elemento neuroanatómico hipotalámico relacionado con la producción de hormona antidiurética y su asociación como una vía potencial de invasión del COVID-19 al sistema nervioso central, afectando la regulación fisiológica de liberación de hormonas relacionadas con la homeostásis del sodio. También se vincula el neurotropismo de este virus al asociarse con el nervio olfatorio, una evaginación del cerebro en la que se altera su funcionalidad por generación de disosmia entre otras características neurosemiológicas. Se plantea la necesidad de advertir a los profesionales de la salud en general y a los neurólogos en especial, sobre las potenciales alteraciones neurológicas relacionadas con esta pandemia antes y después del contagio de este virus e implementar una prueba olfatoria rápida con ácido acético, incluso antes de otras valoraciones como hipertérmia, tos y cefalalgia.

PALABRAS CLAVE: COVID-19, neurología, órgano vascular de la lámina terminal, sodio.

\section{INTRODUCCIÓN}

Por la pandemia de curso actual en la mayoría de nuestro planeta, el COVID-19 se ha caracterizado especialmente por afectar a la mayoría de la población en su sistema respiratorio con consecuencias fatales (Mazza et al., 2020) por generar síndrome respiratorio agudo severo -SARS- además hay pruebas que demuestran su existencia en el líquido cerebroespinal de personas que han sido contagiadas por este virus, lo que se ha confirmado por secuenciación de genes, indicando que hay invasión directa del sistema nervioso, no necesariamente afectándolo a través de la respuesta inmune (Zhou et al., 2020). Estas propiedades neuroinvasivas del COVID19 (Zhou et al.), involucran a la molécula angiotensina II, un correceptor del coronavirus-19 (South et al.,
2020). También se sabe que los pacientes están dando sintomatología de alteración olfativa-además de gustativa- ya que al inhalarse pequeñas gotas con el virus, debidas a mecanismo de tos, habla susurrante o estornudo, ingresan por la vía respiratoria hacia la porción superior de las fosas nasales y luego a través de los tractos y estrías olfatorias, hacia la corteza piriforme (Duque Parra et al., 2016), por lo que es importante obtener información precisa sobre su progresión, dado que muchos pacientes tienen síntomas leves o nulos, de allí que sea importante relacionar rápidamente cualquier síntoma (Gautier \& Ravussin, 2020) para actuar más prontamente en el tratamiento que lleve a intentar preservar la vida de estos pacientes.

\footnotetext{
${ }^{1}$ Facultad de Ciencias de la salud. Universidad de Manizales, Manizales, Colombia.

${ }^{2}$ Departamento de Ciencias Básicas. Universidad de Caldas, Manizales, Colombia.

${ }^{3}$ Grupo Neurociencia de Caldas. Universidad de Caldas, Colombia.

${ }^{4}$ Departamento Básico Clínico. Universidad de Caldas, Manizales, Colombia.
} 
DUQUE PARRA, J. E.; DUQUE MONTOYA, D. \& PELÁEZ, F. J. C. EI COVID-19 también afecta el sistema nervioso por una de sus compuertas: el órgano vascular de la lámina terminal y el nervio olfatorio. Alerta neurológica, prueba de disosmia o anosmia puede ayudar a un diagnóstico rápido. Int. J. Odontostomat., 14(3):285-287, 2020.

\section{DISCUSIÓN}

La ingesta de agua es una de las respuestas fisiológicas básicas necesarias para mantener la vida de los seres humanos, que si se afecta puede comprometerla (Gautier \& Ravussin). Una de las partes de nuestro sistema nervioso central que monitorea esta función, es particularmente uno de sus órganos circunventriculares: el órgano vascular de la lámina terminal (organum vasculosum laminae terminalis) (Gizowski \& Bourque, 2018), el cual posee capilares fenestrados (Kleine, 2015), lo que representa el punto clave para su invasión, puesto que allí no hay barrera hematoencefálica que proteja al encéfalo. De esta manera, el virus puede afectar la modulación de la presión sanguínea como se ha demostrado en animales de experimentación (García Hernández-Abad \& Ruiz-Mayor, 2017), ya que allí hay neuronas que emiten axones a los núcleos supraóptico y paraventricular del hipotálamo, facilitando la despolarización de las neuronas que producen hormona antidiurética (Mtui et al., 2016). Esto llevaría al aumento de la hipertonicidad sanguínea (Rondon-Berrios \& Berl, 2019), comprometiendo la funcionalidad total del sistema nervioso en vista que los mecanismos de despolarización nerviosa dependen fundamentalmente de la concentración del sodio circulante (Navarro et al., 2020), sea sobre motoneuronas, neuronas sensitivas o interneuronas, además de comprometer otros sistemas corporales como el cardiovascular, pues se han encontrado pacientes que desarrollan lesiones cardíacas agudas durante esta enfermedad (Bansal, 2020). Por lo tanto, los pacientes tratados por la pandemia del COVID-19, presentan y presentarán alteraciones neurológicas múltiples que deben alertar a los neurólogos para su tratamiento, a la par de alteraciones neumológicas durante la evaluación y tratamiento, una de ellas que se está implementando en países asiáticos como lo es hacer oler al paciente una solución con vinagre, incluso por personas que no tienen mayor entrenamiento clínico, y ante la respuesta de alteración en la olfacción, sospechar inmediatamente que es un paciente con COVID-19, quizá sin síntomas de fiebre, tos u otras.

\section{CONCLUSIÓN}

El sistema nervioso de las personas afectadas por el COVID-19 se afectará, involucrando otros sistemas orgánicos, por lo que, en las valoraciones semiológicas de personas afectadas por este virus, se debe hacer un seguimiento neuro semiológico durante la valoración del caso con pruebas olfativas, como oler vinagre para hacer diagnóstico previo rápido y también posterior al problema neumológico generado por este agente infeccioso, lo que involucrará secuelas disfuncionales neurológicas y multisistémicas, algunas de ellas aún no descritas.

DUQUE PARRA, J. E.; DUQUE MONTOYA, D. \& PELÁEZ, F. J. C. COVID-19 also affects the nervous system by one of its gates: the vascular organ of lamina terminalis and the olfactory nerve. Neurological alert, dysosmia or anosmia test can help to a quick diagnosis. Int. J. Odontostomat., 14(3):285-287, 2020.

ABSTRACT: A brief review of the vascular organ of lamina terminalis (organum vasculosum laminae terminalis) and the olfactory nerve is presented, the former is an hypothalamic neuroanatomical element related to the production of antidiuretic hormone and its association as a potential route of invasion of the COVID-19 to the central nervous system, affecting the physiological regulation of hormone release related to sodium homeostasis. The neurotropism of this virus is also linked when it is associated with the olfactory nerve, an evagination of the brain in which its functionality is altered by the generation of dysosmia, among other neurosemiological characteristics. The need arises to warn health professionals in general and neurologists in particular, about the potential neurological disorders related to this pandemic before and after the spread of this virus and to implement a rapid olfactory test with acetic acid, even before other evaluations such as hyperthermia, cough and headache.

KEY WORDS: COVID-19, neurology, vascular organ of lamina terminalis, sodium.

\section{REFERENCIAS BIBLIOGRÁFICAS}

Bansal, M. Cardiovascular disease and COVID-19. Diabetes Metab. Syndr., 14(3):247-50, 2020.

Duque Parra, J. E.; Marín Arias, J. F. \& Peláez Cortés, F. J. C. Olfactory stria in the human being and proposal for inclusion in Terminologia Anatomica of olfactory intermediate stria. Int. J. Morphol., 34(4):1411-3, 2016.

García Hernández-Abad, L. \& Ruiz-Mayor, M. L. Comportamiento de los órganos circunventriculares en la hipertensión sistémica. Revisión. Majorensis, 13:69-79, 2017.

Gautier, J. F. \& Ravussin, Y. A new symptom of COVID-19: loss of taste and smell. Obesity (Silver Spring), 2020. https://doi.org/ 10.1002/oby.22809

Gizowski, C. \& Bourque, C. W. The neural basis of homeostatic and anticipatory thirst. Nat. Rev. Nephrol., 14(1):11-25, 2018.

Kleine, T. O. Cellular immune surveillance of central nervous system bypasses blood-brain barrier and blood-cerebrospinal-fluid 
DUQUE PARRA, J. E.; DUQUE MONTOYA, D. \& PELÁEZ, F. J. C. El COVID-19 también afecta el sistema nervioso por una de sus compuertas: el órgano vascular de la lámina terminal y el nervio olfatorio. Alerta neurológica, prueba de disosmia o anosmia puede ayudar a un diagnóstico rápido. Int. J. Odontostomat., 14(3):285-287, 2020.

barrier: revealed with the New Marburg cerebrospinal-fluid model in healthy humans. Cytometry A, 87(3):227-43, 2015.

Mazza, S.; Sorce, A.; Peyvandi, F.; Vecchi, M. \& Caprioli, F. A fatal case of COVID-19 pneumonia occurring in a patient with severe acute ulcerative colitis. Gut, 2020. DOI: doi: https://doi.org/ 10.1136/gutjnl-2020-321183

Mtui, E.; Gruener, G. \& Dockery, P. Fitzgerald. Neuroanatomía Clínica y Neurociencia. Barcelona, Elsevier, 2016. pp.253-9.

Navarro, M. A.; Salari, A.; Lin, J. L.; Cowan, L. M.; Penington, N. J.; Milescu, M. \& Milescu, L. S. Sodium channels implement amolecular leaky integrator that detects action potentials and regulates neuronal firing. Elife, 9:e54940, 2020.

Rondon-Berrios, H. \& Berl, T. Physiology and pathophysiology of water homeostasis. En: Peri, A.; Thompson, C. J. \& Verbalis, J. G. (Eds.). Disorders of Fluid and Electrolyte Metabolism. Focus on Hyponatremia. Front. Horm. Res. Vol. 52. Basel, Karger, 2019. pp.8-23.

South, A. M.; Diz, D. \& Chappell, M. C. COVID-19, ACE2 and the cardiovascular consequences. Am. J. Physiol. Heart Circ. Physiol., 2020. https://doi.org/10.1152/ajpheart.00217.2020

Zhou, L.; Zhang, M.; Wang, J. \& Gao, J. Sars-Cov-2: Underestimated damage to nervous system. Travel Med. Infect. Dis., 24:101642, 2020.
Dirección para correspondencia:

Jorge Eduardo Duque Parra

Departamento de Ciencias Básicas

Universidad de Caldas

Manizales

COLOMBIA

ORCID https://orcid.org/0000-0002-1432-6381

E mail: jduqueparra@yahoo.com.mx

Recibido : 07-04-2020

Aceptado: 08-04-2020 